\title{
Implementation of Dynamic Threshold Method for Human Motion Detection in Video surveillance application \\ Ms. Pritee Gupta ${ }^{1}$ Dr. Yashpal Singh ${ }^{2}$
}

${ }^{1}$ Ph.D Student, Bhagwant University, Ajmer \& Assistant Professor, CSED, I.T.S Engineering College, Greater Noida, India

${ }^{2}$ Associate Professor \& Head, IT Department, B.I.E.T, Jhansi, India

\begin{abstract}
Detection of moving objects in video streams is the first relevant step of information extraction in many computer vision applications. Aside from the intrinsic usefulness of being able to segment video streams into moving and background components, detecting moving objects provides a focus of attention for recognition, classification, and activity analysis, making these later steps more efficient. This paper implemented a method to detect moving object based on background subtraction. First of all, we establish a reliable background updating model based on statistical and use a dynamic optimization threshold method to obtain a more complete moving object. The moving human bodies are accurately and reliably detected. The experiment results show that the proposed method runs quickly, accurately and fits for the real-time detection.
\end{abstract}

\section{Keywords:}

Frame separation; Background subtraction; Dynamic threshold; Motion detection.

\section{Council for Innovative Research}

Peer Review Research Publishing System

\section{Journal: INTERNATION JOURNAL OF COMPUTERS AND TECHNOLOGY}

Vol. 13, No. 8

editorijctonline@gmail.com

www.ijctonline.com, www.cirworld.com 


\section{INTRODUCTION}

Surveillance is the monitoring of behavior. Systems surveillance is the process of monitoring the behavior of people, objects or processes within systems for conformity to expected or desired norms in trusted systems for security or social control [1]. The word surveillance is commonly used to describe observation from a distance by means of electronic equipment or other technological means.

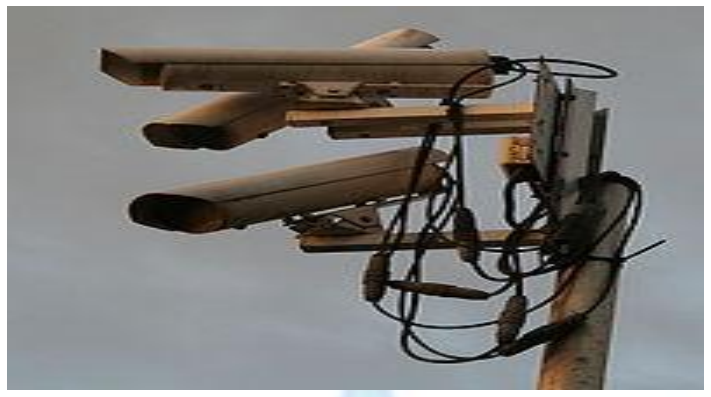

Fig. 1 Example of CCTV camera

At a basic level, computers are a surveillance target because large amounts of personal information are stored on them. Anyone who can access or remove a computer can retrieve information. If someone is able to install software on a computer system, they can turn the computer into a surveillance device. CCTV is a collection of video cameras used for video surveillance. CCTV is generally used in areas where there is an increased need for security, such as banks, airports and town centers. A basic CCTV system comprises of the Camera, lens and power supply. Recording device, VCR or a digital video recorder and monitor. Closed-circuit television (CCTV) is the use of video cameras to transmit a signal to a specific place, on a limited set of monitors. The main tasks in visual surveillance systems include motion detection, object classification, tracking. Our focus here is on the detection phase of a general visual surveillance system using static cameras. The detection of moving objects in video streams is the first relevant step of information extraction in many computer vision applications. Aside from the intrinsic usefulness of being able to segment video streams into foreground and background components, detecting moving objects provides a focus of attention for recognition, classification, and activity analysis, making these later steps more efficient, since only moving pixels need be considered [2].The usual approach to moving object detection is through background subtraction, that consists in maintaining an up-to date model of the background and detecting moving objects as those that deviate from such a model. Compared to other approaches, such as optical flow [3], this approach is computationally affordable for real-time applications. The background image is not fixed but must adapt to: Illumination changes, sudden (such as clouds) ,Motion changes ,camera oscillations, highfrequencies background objects (such as tree branches, sea waves, and similar) Changes in the background geometry.

\section{BACKGROUND SUBTRACTION}

Identifying moving objects from a video sequence is a fundamental and critical task in many computer-vision applications. A common approach is to perform background subtraction, which identifies moving objects from the portion of a video frame that differs significantly from a background model. There are many challenges in developing a good background subtraction algorithm. First, it must be robust against changes in illumination. Second, it should avoid detecting nonstationary background objects such as moving leaves, rain, snow, and shadows cast by moving objects [4]. Some of the well-known issues in background maintenance that will be specifically addressed in the sequel include:

> Light changes: The background model should adapt to gradual illumination changes.

> Moving background: The background model should include changing background that is not of interest for visual surveillance, such as waving trees.

$>$ Cast shadows: The background model should include the shadow cast by moving objects that apparently behaves itself moving, in order to have a more accurate detection of the moving objects shape.

$>$ Bootstrapping: The background model should be properly set up even in the absence of a complete and static (free of moving objects) training set at the beginning of the sequence.

$>$ Camouflage: Moving objects should be detected even if their chromatic features are similar to those of the background model.

$>$ This paper implemented a reliable background updating model based on statistical and use a dynamic optimization threshold method to obtain a more complete moving object.

\section{PROPOSED WORK IMPLEMENTATION}

In this work the aim is to build such a surveillance system, which can detect motion even if the moving background, gradual illumination variations and camouflage and shadow into the background, thus achieves robust detection for different types of videos taken with stationary cameras. To fulfill this aim, strong computing software called Mat lab is used. Mat lab provides image Acquisition and Image Processing Toolboxes which facilitate us in creating a good code. 


\section{Frame Separation}

Frame processing is the first step in the background subtraction algorithm, the purpose of this step is to prepare the modified video frames by removing noise and unwanted object's in the frame in order to increase the amount of information gained from the frame and the sensitivity of the algorithm. Preprocessing is a process of collecting simple image processing tasks that change the raw input video info a format. This can be processed by subsequent steps. Preprocessing of the video is necessary to improve the detection of moving object's For example, by spatial and temporal smoothing, snow as moving leaves on a tree, can be removed by morphological processing of the frames after the identification of the moving object's as shown in figure.
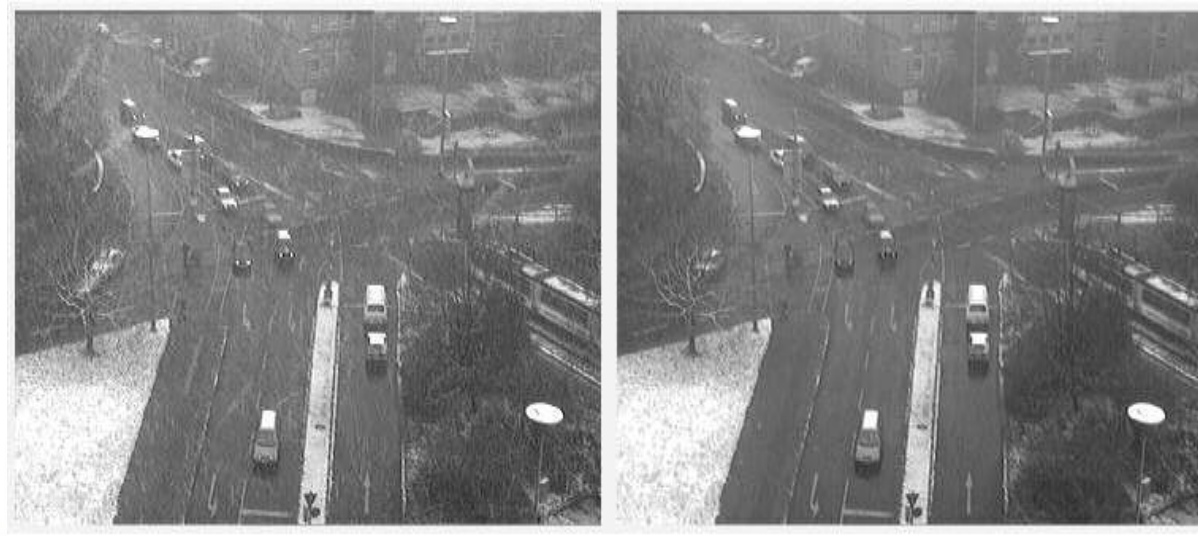

Fig. 2: Pre processing of input video frames

Another key issue in pre processing is the data format used by the particular background subtraction algorithm. Most of the algorithm handles luminance intensity, which is one scalar value per each pixel, however, color image, in either RGB or HSV color space, is becoming more popular in the background subtraction algorithms.

\section{Coding for Frame Separation:}

file=aviinfo('movie1.avi');

frm_cnt=file.NumFrames

str2='.bmp'

$\mathrm{h}=$ waitbar(0,'Please wait...');

for $\mathrm{i}=1$ :frm_cnt

frm(i)=aviread(filename,i);

frm_name=frame2im(frm(i));

frm_name=rgb2gray(frm_name);

filename1=strcat(strcat(num2str(i)),str2);

imwrite(frm_name,filename1);

waitbar(i/frm_cnt,h)

end

close(h)

\section{BACKGROUND MODELING AND UPDATE}

The basic scheme of background subtraction is to subtract the image from a reference image that models the background scene. Typically, the Background modeling constructs a reference image representing the background. Threshold selection determines appropriate threshold values used in the subtraction operation to obtain a desired detection rate. Subtraction operation or pixel classification classifies the type of a given pixel, i.e., the pixel is the part of background (including ordinary background and shaded background), or it is a moving object [5]. 


\section{Background Modeling}

In the background training process, the reference background image and some parameters associated with normalization are computed over a number of static background frames. The background is modeled statistically on a pixel by pixel basis. A pixel is modeled by a 4-tuple < Ei; si; ai; bi > where Ei is the expected color value, si is the standard deviation of color value which is defined in ai is the variation of the brightness distortion, and bi is the variation of the chromaticity distortion of the ith pixel. $\mathrm{Ei}$, ai and bi are defined explicitly later in this section. The expected color value of pixel $\mathrm{i}$ is given by

$$
\begin{aligned}
& E_{i}=\left[\mu_{R}(i), \mu_{G}(i), \mu_{B}(i)\right] \\
& \text { Where } \mu_{R}(i) \mu_{G}(i) \text { and } \mu_{B}(i)
\end{aligned}
$$

are the arithmetic means of the ith pixel's red, green, blue values computed over $\mathrm{N}$ background frames. So far, we have defined $\mathrm{Ei}$ and si. We also discussed about balancing color bands by rescaling the color values by the pixel variation factors (si). Thus the brightness distortion and the chromaticity.

\section{Background Update}

For the background model can better adapt to light changes, the background needs to be updated in real time, so as to accurately extract the moving object. In this paper, the update algorithm is as follows: In detection of the moving object, the pixels judged as belonging to the moving object maintain the original background gray values, not be updated. For the pixels which are judged to be the background, we update the background model according to following rules:

$$
B_{k+1}(x, y)=\beta B_{k}(x, y)+(1-\beta) F_{k}(x, y)
$$

Where $f 3 E(0,1)$ is update coefficient, $m$ this paper $f 3=0.004 . F k(x, y)$ is the pixel gray value in the current frame. $B k(x, y)$ and $B k+1(x, y)$ are respectively the Background value of the current frame and the next frame. As the camera is fixed, the background model can remain relatively stable in the long period of time. Using this method can effectively avoid the unexpected phenomenon of the Background, such as the sudden appearance of something in the background which is not included in the original background. Moreover by the update of pixel gray value of the background, the impact brought by light, weather and other changes in the external environment can be effectively adapted.

\section{Moving Object Extraction}

After the background image $B(x, y)$ is obtained, subtract the background Image $B(x, y)$ from the current frame $F k$ ( $x$, y). If the pixel difference is greater than the set threshold $\mathrm{T}$, then determines that the pixels appear in the moving object, otherwise, as the background pixels. The moving object can be detected after threshold operation. Its expression is as follows:

$$
D_{k}(x, y)= \begin{cases}1 & \left|F_{k}(x, y)-B_{k-1}(x, y)\right|>T \\ 0 & \text { others }\end{cases}
$$

Where $D k(x, y)$ is the binary image of differential results. $T$ is gray-scale threshold; its size determines the accuracy of object identification. As in the algorithm $T$ is a fixed value, only for an ideal situation, is not suitable for complex environment with lighting changes. Therefore, this paper implemented the dynamic threshold method, we dynamically changes the threshold value according to the lighting changes of the two images obtained. On this basis, add a dynamic threshold!' $1 T$ to the above algorithm. Its mathematical expression is as follows:

$$
\begin{gathered}
\Delta T=\lambda \cdot \frac{1}{M \times N} \sum_{t=0}^{N-1} \sum_{j=0}^{M-1}|F(i, j)-B(i, j)| \\
D_{k}(x, y)= \begin{cases}1 & \left|F_{k}(x, y)-B_{k-1}(x, y)\right|>T+\Delta T \\
0 & \text { others }\end{cases}
\end{gathered}
$$

Where $A$ is the inhibitory coefficient, set it to a value according to the requirements of practical applications, and the reference values is $2 . \mathrm{M} \times \mathrm{N}$ is the size of each image to deal with. $\mathrm{M} \times \mathrm{N}$ numerical results indicate the number of pixels in detection region. This method can effectively suppress the impact of light changes ([6], [7], [8]). 


\section{RESUL AND DISCUSSION}

Several points for moving object detection using the given approach have been produced for input image .

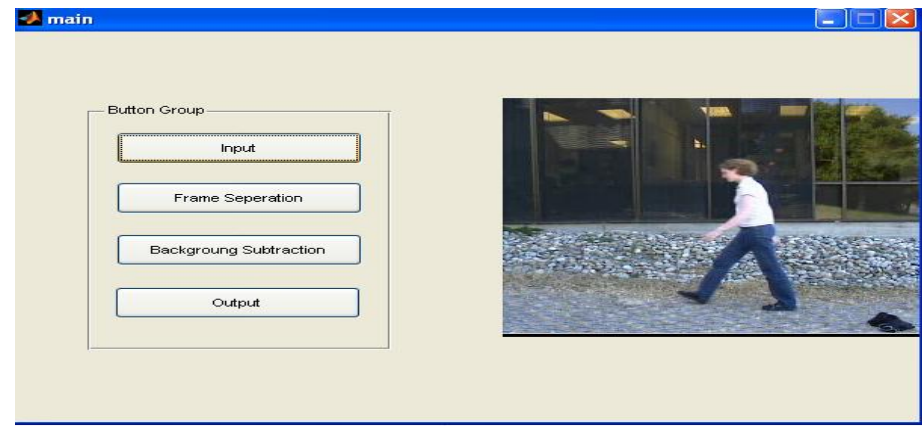

Fig. 3: Input video

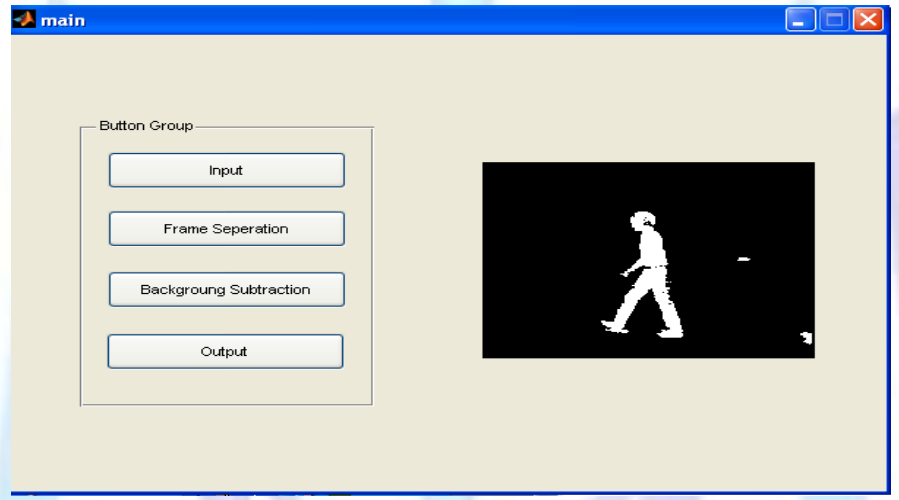

Fig.4: Output video (Moving Person is perfectly detected)

Values for the distance thresholds should be chosen such that High values for allow to limit selectivity in the update of the background model during the calibration phase, enabling the inclusion into the initial background model of several observed pixel intensity variations. Lower values for should be chosen to obtain a more accurate background model in the online phase by applying the detection mask, we can observe that the object is almost perfectly detected, despite his camouflage and moving background.

\section{VI.CONCLUSION AND ONGOING WORK}

In this paper, a real-time and accurate new method for detecting moving human body is implemented based on background subtraction. In cognizance of the shortcomings and deficiencies in the traditional method of object detection, we establish reliable background model, use dynamic threshold method to detect moving object and update the background in real time. Experiments show that the algorithm is fast and simple, able to detect moving human body better and it has a broad applicability. 


\section{REFERENCES}

[1] Du-Ming Tsai and Shia- Chih Lai, "Independent Component Analysis Based Background Subtraction for Indoor Surveillance," Image Processing, IEEE Transactions on Volume 18, Issue 1, Jan. 2009 Page(s):158 - 16.

[2] K. Kinoshita, M. Enokidani, M. Izumida and K. Murakami, "Tracking of a Moving Object Using One-Dimensional Optical Flow with a Rotating Observer," Control, Automation, Robotics and Vision, 2006. ICARCV '06. 9th International Conference on 5-8 Dec. 2006 Page(s): 1 - 6.

[3] M. Dimitrijevic, "Human body pose detection using Bayesian spatiotemporal Templates," 2007 International Conference on Intelligent and Advanced Systems, 2008, pp.764-9.

[4] Niu Lianqiang and Nan Jiang, "A moving objects detection algorithm based on improved background subtraction," Intelligent Systems Design and Applications, 2008. ISDA '08. Eighth International Conference on Volume 3, 26-28 Nov. 2008 Page(s):604 - 60.

[5] N.J. Bauer and P.N. Pathirana, "Object focused simultaneous estimation of optical flow and state dynamics," Intelligent Sensors, Sensor Networks and Information Processing, 2008. ISSNIP 2008. International Conference on 15-18 Dec. 2008 Page(s):6l- 66 .

[6] R. T. Collins, A. J. Lipton, T. Kanade, H. Fujiyoshi, D. Duggins, Y. Tsin, D. Tolliver, N. Enomoto, O. Hasegawa, P. Burt, and L. Wixson, "A system for video surveillance and monitoring," Tech. Rep. CMU-RI-TR-00-12, The Robotics Inst., Carnegie Mellon Univ., Pittsburgh, PA, 2000.

[7] Tao Jianguo, Yu Changhong, "Real-Time Detection and Tracking of Moving Object," Intelligent Information Technology Application, 2008. UTA '08. Second International Symposium on Volume 2, 20-22 Dec. 2008 Page(s):860 - 863.

[8] Zhen Tang and Zhenjiang Miao, "Fast Background Subtraction and Shadow Elimination Using hnproved Gaussian Mixture Model," Haptic, Audio and Visual Environments and Garnes, 2007. HAVE 2007. IEEE International Workshop on 12-14 Oct. 2007 Page(s):38 - 41.

\section{Author' biography with Photo}

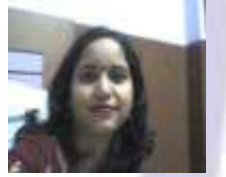

Pritee Gupta was born on March 23rd, 1979, in Lucknow, India. She is B.Tech, M.Tech. \& Pursuing $\mathrm{PhD}$ in Computer Science \& Engineering. She has 12 years of teaching experience in various Engg. Colleges of India. She has published more than 10 papers in national and international technical journals. Her current research interests include computer vision, neural networks and image processing. She is a member of the International Neural Network Society, IAENG and the Computer society of India.

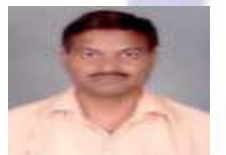

Dr. Yashpal Singh is Associate Professor and Head, Department of Information technology in Bundelkhand Institute of Engineering and Technology Jhansi (INDIA). He has published more than 20 papers in national and international technical journals. His current research interests include OOPS, Data structure, Image processing, Neural Networks. He is a member of the International Association of Engineers (IAENG), Hong Kong, and the Computer society of India. 